\title{
Gold Nanoparticles Promote Proliferation of Human Periodontal Ligament Stem Cells and Have Limited Effects on Cells Differentiation
}

\author{
Chen Li, ${ }^{1}$ Zhuoquan $\mathrm{Li}^{2}{ }^{2}$ Yan Wang, ${ }^{1}$ and Hongwei Liu ${ }^{1}$ \\ ${ }^{1}$ Department of Periodontics, School \& Hospital of Stomatology, Tongji University, Shanghai Engineering Research Center of \\ Tooth Restoration and Regeneration, Shanghai 200072, China \\ ${ }^{2}$ The Institute for Biomedical Engineering and Nanoscience, School of Medicine, Tongji University, \\ 67 Chifeng Road, Shanghai 200092, China \\ Correspondence should be addressed to Hongwei Liu; hwliu@tongji.edu.cn
}

Received 23 February 2016; Revised 26 May 2016; Accepted 29 June 2016

Academic Editor: Jorge Pérez-Juste

Copyright (C) 2016 Chen Li et al. This is an open access article distributed under the Creative Commons Attribution License, which permits unrestricted use, distribution, and reproduction in any medium, provided the original work is properly cited.

Gold nanoparticles (AuNPs) had been widely applied in the practice and advancement of chemistry, biology, and medicine due to facility of synthesis and versatility in surface functionalization. Recent studies had shown that AuNPs can be applied to cells, affecting cellular physiological processes such as proliferation and differentiation. In this study, four diameters of AuNPs (20, 40, 60 , and $80 \mathrm{~nm}$ ) were cocultured with human periodontal ligament cells (hPDLCs) at six different concentrations. The optimal size and concentration of AuNPs were selected to treat human periodontal ligament stem cells (hPDLSCs) to evaluate proliferation. Moreover, the influence of AuNPs on multiple differentiation capacity of hPDLSCs was clarified. The results revealed that AuNPs $(60 \mathrm{~nm}, 56 \mu \mathrm{M})$ can effectively promote the proliferation of hPDLCs/hPDLSCs in vitro, slightly enhance osteoblastic differentiation, and have no effect on adipogenic differentiation. In addition, the expression of COL-1, Runx2, BSP, and OCN was upregulated in the presence of AuNPs $(60 \mathrm{~nm}, 56 \mu \mathrm{M})$. These results indicated that AuNPs $(60 \mathrm{~nm}, 56 \mu \mathrm{M})$ can effectively promote the proliferation of hPDLCs/hPDLSCs and have no significant effect on the differentiation of hPDLSCs. These results provide an insight on the advantage of implementing of AuNPs on hPDLSCs culture and expose the influence of these materials on periodontal tissue engineering.

\section{Introduction}

The periodontal ligament (PDL) is a soft connective tissue embedded between cementum and inner wall of the alveolar bone socket that function as an anchor and nourish the teeth. One of the periodontal diseases consists of the inflammation altering the tissues, surrounding and supporting teeth, initiating progressive bone loss around it, which results in mobility of teeth and eventually their avulsion if treatment is not pursued [1]. Neither periodontal initial therapy such as supragingival scaling and root planning (SRP) nor guided tissue regeneration (GTR) can lead to the defect of periodontal tissue to obtain complete regeneration. In recent years, with the rapid development of tissue engineering, periodontal tissue regeneration has opened up a new way for the treatment of periodontal disease. Periodontal ligament stem cells (PDLSCs) derived from periodontal ligament was able to differentiate into cementoblast-like cells, adipocytes, and collagen-forming cells in vitro and potentiate generating cementum/PDL-like tissue in vivo [2]. As one of the three elements of periodontal tissue engineering, PDLSCs had been widely used in tissue reconstruction, not only for replacing destroyed periodontium in animal and human models [2] but also for other applications such as the formation of bone around prosthetic implants [3] and even plastic reconstruction [4]. However, its application in tissue engineering was limited because there were few PDLSCs in the periodontal ligament [5]. The former results indicated that the capability 
of differentiation of cultured mesenchymal stem cells (MSCs) was descending during passage $[6,7]$. It is considered a challenge to proliferate PDLSCs rapidly in vitro while maintaining the stem cells biology characteristics in tissue engineering.

Gold nanoparticles (AuNPs) belong to a kind of nanomaterial that has shown advanced biocompatibility, low cytotoxicity, and strong oxidation resistance, which can be further controlled by engineering and altering them to an optimal extent through controllable size and shape capabilities and the possibility of combining them with many varieties of biological macromolecules. Without influence on biological characteristics, AuNPs had been widely employed in bioengineering biomedicine including drug delivery tactics [8], diagnostic imaging [9], antibody labeling, and targeting [10, 11]. AuNPs were able to traverse biological barriers and eventually enter the cell nuclei to alter physiological processes such as proliferation and differentiation [12]. However, there were contradictory results concerning the toxicity of AuNPs [13]. Research results showed that certain size and concentration of AuNPs have a promoting effect on cells proliferation, and it was confirmed that the cytotoxicity of AuNPs is size-, concentration-, and time-dependent [14].

The effect of AuNPs on cell proliferation and differentiation was studied; however, the effect of AuNPs on proliferation and differentiation of human periodontal ligament stem cells (hPDLSCs) has not been reported. In the present study, we investigated the effect of four sizes of AuNPs at six different concentrations on the proliferation of human periodontal ligament cells (hPDLCs). The optimal size and concentration of gold nanoparticles (AuNPs) were treated with hPDLSCs to study the proliferation. Positive rate of STRO-1 and CD146 in hPDLSCs treated with AuNPs was measured with FCM. In addition, in order to unveil the differentiation of hPDLSCs treated with AuNPs, Alkaline Phosphatase (ALP) activities, ALP Staining, Alizarin red staining, and Oil red $\mathrm{O}$ staining have been investigated. Moreover, changes in the expression of osteogenic genes (COL-1, Runx2, BSP, and OCN) were evaluated.

\section{Materials and Methods}

2.1. Materials. Fetal bovine serum (Hyclone, USA), alphaminimum essential medium ( $\alpha$-MEM) (Hyclone, USA), MTS (Promega, USA), Trizol Reagent kit (Invitrogen, USA), ALP activity kit (Sigma, USA), real-time PCR kit (Invitrogen, USA), disodium $\beta$-glycerophosphate ( $\beta$-GP) (Sigma, USA), ascorbic acid (Sigma, USA), and dexamethasone (Sigma, USA) were used in this study. Four sizes of AuNPs (20, 40, 60 , and $80 \mathrm{~nm}$ ) were purchased from BBI and were characterized by UV-vis spectroscopy and zeta sizer and potential (see Figure s1 in Supplementary Material available online at http://dx.doi.org/10.1155/2016/1431836).

2.2. Cell Culture. Premolars from 12- and 13-year-old teenagers extracted for orthodontic reasons were obtained freshly from the Oral Surgery Department with informed consent. Teeth were rinsed three times with PBS supplemented with Antibiotic-Antimycotic. Periodontal ligament tissue in the middle third of the root was carefully removed with a surgical scalpel. Tissue was minced and enzymatically digested using type I collagenase ( $1 \%$, Gibco) and Dispase II (2.5\%, Roche, Germany) for one hour at $37^{\circ} \mathrm{C}$. A suspended cell solution passed through $70 \mu \mathrm{m}$ cell strainer to prepare single cell suspension. The cells were centrifuged and rinsed with PBS. The cell pellet was resuspended and cultured in alpha-modified minimum essential medium ( $\alpha$-MEM, Gibco) with $10 \%$ FBS (Gibco) and $1 \%$ penicillin-streptomycin in a humidified atmosphere $\left(37^{\circ} \mathrm{C}, 5 \% \mathrm{CO}_{2}\right)$, until the cells proliferated and reached $70-80 \%$ confluence. The hPDLCs were identified by cellular morphology and marker antibodies (Figure s2). FCM was employed to sort hPDLSCs where morphology and osteogenic and adipogenic differentiation were on preliminary identification (Figure s3).

2.3. MTS Assay. The hPDLCs of the fourth passage were seeded into 96-well plates at a density of 1000 cells per well and incubated overnight. The medium was removed carefully and cells were washed with PBS. Four diameters of AuNPs $(20,40,60$, and $80 \mathrm{~nm}$ ) were diluted with fresh serum-free $\alpha$-MEM medium and cultured with hPDLCs at $1.75,3.5,7,14,28$, and $56 \mu \mathrm{M}$, respectively. Two hours later, the medium was aspirated and replaced with $\alpha$-MEM medium containing $10 \%$ FBS. Cells without AuNPs were used as negative control; wells without AuNPs or cells were used as blank for spectrophotometric readings. $20 \mu \mathrm{L}$ of MTS (Promega, USA) was added to each well at 6, 12, 24, 48, and 72 hours. After incubation at $37^{\circ} \mathrm{C}$ for two hours, the optical density value (OD values) for each well was measured spectrophotometrically at $490 \mathrm{~nm}$, and the assay was repeated five times.

The best combination of size and concentration of AuNPs to promote hPDLCs proliferation was used to treat hPDLSCs. hPDLSCs without AuNPs were used as negative control. Cell culture and MTS assay were described above.

2.4. Immunofluorescence. hPDLCs were identified by immunofluorescence with vimentin and cytokeratin. Cells were washed with PBS twice, fixed with $4 \%$ paraformaldehyde for $15 \mathrm{~min}$, and then permeabilized with $0.1 \%$ Triton X-100 for five min and blocked by $3 \%$ bovine serum albumin for $30 \mathrm{~min}$ at room temperature. The coverslips were stained with primary antibodies overnight at $4^{\circ} \mathrm{C}$ followed by incubation with secondary antibody (red) (Invitrogen) for $30 \mathrm{~min}$ at $37^{\circ} \mathrm{C}$. The nuclei were stained using DAPI (blue) $(0.5 \mu \mathrm{g} / \mathrm{mL}$, CST, USA) for ten min. Cells were washed three times with PBS in a dark chamber. The coverslips were washed as described above, inverted, mounted on slides, and examined in a Nikon ECLIPSE Ti-S microscopy.

2.5. Flow Cytometric Analysis and Sorting. STRO-1 ${ }^{+}$hPDLCs were sorted by flow cytometry (FCM) and identified preliminarily for its stem cell characteristics through plate clone formation assay, multidirectional induction as osteoblastic, and adipocytic differentiation.

hPDLSCs were cultured with $60 \mathrm{~nm} 56 \mu \mathrm{M}$ AuNPs, and the method was the same as above. After incubation at $37^{\circ} \mathrm{C}$ for 72 hours, cells were moved into the flow tube at density of $1 \times 10^{6} / \mathrm{mL}$, setting control/FITC and ISO control group. 
After fixed with $4 \%$ paraformaldehyde for $15 \mathrm{~min}$, cells were incubated with antibody of CD146 and STRO-1, respectively, and the positive expression rates of STRO-1 and CD146 were detected by FCM.

2.6. Alkaline Phosphatase Staining. hPDLSCs were cultured in 24 -well plates at a density of $2 \times 10^{4}$ cells/well. After incubation overnight, the medium was removed and cells were washed with PBS. Cells were cultured with serum-free $\alpha$ MEM medium containing $60 \mathrm{~nm} 56 \mu \mathrm{M}$ AuNPs for three hours. The culture medium was substituted with $\alpha$-MEM medium with $10 \%$ FBS. When $60-70 \%$ of the cells fused, medium was changed to $\alpha$-MEM supplemented with $10 \%$ FBS, $10 \mathrm{mM}$ disodium $\beta$-glycerophosphate $(\beta$-GP), $0.15 \mathrm{mM}$ ascorbic acid, and $10 \mathrm{nM}$ dexamethasone. The medium was changed every two days. Cells incubated with osteogenic inducers without AuNPs were positive controls. Cells incubated with normal medium without AuNPs were blank controls. At 4, 7, and 10 days, the plates were washed three times with ice-cold PBS and then fixed with $4 \%$ paraformaldehyde for $20 \mathrm{~min}$. Finally, the samples were stained by using the $\mathrm{BCIP/NBT}$ alkaline phosphatase staining kit following the manufacturer's instructions.

2.7. Alkaline Phosphatase Activity Assay. hPDLSCs cultured with AuNPs were described above. At 4, 7, and 10 days, cells were washed twice with ice-cold PBS and harvested using $500 \mu \mathrm{L} /$ well of NP40. $80 \mu \mathrm{L}$ solution and $80 \mu \mathrm{L}$ chromogenic agent were added to the wells of the culture plates. After incubation in $37^{\circ} \mathrm{C}$ for two hours, the absorbance was measured spectrophotometrically at $405 \mathrm{~nm}$, and the assay was repeated four times. Protein concentration was measured by using the Bradford protein assay kit following the manufacturer's instructions. Alkaline phosphatase activity was determined using the alkaline phosphatase assay kit. All results were normalized to the protein content of each sample.

2.8. Alizarin Red Staining. hPDLSCs cultured with AuNPs method were the same as above. After osteoblastic induction for 28 days, the plates were washed three times with ice-cold PBS and then fixed with $4 \%$ paraformaldehyde for $20 \mathrm{~min}$. The fixed cells were washed twice with PBS and stained with Alizarin red S for five min at room temperature. The calcified deposits shown in red were then photographed.

2.9. Oil Red O Staining. The adipogenic differentiation of hPDLSCs in the presence of AuNPs was determined by an Oil Red O staining assay. hPDLSCs cultured with AuNPs method were the same as above. After treated with AuNPs, hPDLSCs were cultured with $\alpha$-MEM medium until $100 \%$ of the cells fused. After adipogenic induction for 24 days, the cells were washed with PBS and they were fixed with $4 \%$ paraformaldehyde for $20 \mathrm{~min}$. The fixed cells were washed twice with PBS and stained with Oil Red O solution for $30 \mathrm{~min}$ at room temperature.

2.10. The Quantification of Alizarin Red Staining and Oil Red O Staining. The number of hPDLSCs in AuNPs treated group and control group was adjusted to be $5.0 \times 10^{3}$ per well in 96-well plate. After osteoblastic induction for 28 days, the plates were washed three times with ice-cold PBS and then fixed with $4 \%$ paraformaldehyde for $20 \mathrm{~min}$. The fixed cells were washed twice with PBS and stained with Alizarin red S for five min at room temperature. Quantification of Alizarin red S staining was performed by elution with $10 \%(\mathrm{w} / \mathrm{v})$ cetylpyridinium chloride (Sigma, USA) for $10 \mathrm{~min}$ and measurement of the absorbance at $570 \mathrm{~nm}$. The relative mineralization rate compared to control group was expressed as a percentage: OD sample/OD control $\times 100 \%(n=3)$.

After adipogenic induction for 24 days, the cells were washed with PBS and they were fixed with $4 \%$ paraformaldehyde for $20 \mathrm{~min}$. The fixed cells were washed twice with PBS and stained with Oil Red $\mathrm{O}$ solution for $30 \mathrm{~min}$ at room temperature. Quantitation of lipid was done by measuring the extent of Oil red $\mathrm{O}$ staining. After removing the staining solution, the dye retained in the cells was eluted into isopropanol (Sigma, USA) and OD540 was determined. The intracellular lipids content of cells was expressed as a percentage: OD sample/OD control $\times 100 \%(n=3)$.

2.11. Real-Time PCR. After treated with and without AuNPs, hPDLSCs were cultured for four days. Total RNA was isolated using the Trizol Reagent following the manufacturer's instructions, and RNA concentration was measured using spectrophotometry (Eppendorf BioPhotometer, Germany). First strand cDNA was developed using the RNA Reverse Transcription (RT) kit following the manufacturer's instructions. PCR was performed using $1 \mu \mathrm{L}$ of the RT products as the template. For the amplification step, 40 cycles were conducted under the following conditions: denaturation at $94^{\circ} \mathrm{C}$ for two min, annealing at $58^{\circ} \mathrm{C}$ for $30 \mathrm{sec}$, and extension at $72^{\circ} \mathrm{C}$ for $45 \mathrm{sec}$. The specific gene primers used in real-time PCR are displayed in Table 1. Real-time polymerase chain reaction (RT-PCR) was used to measure the expression of the osteogenic genes (COL-1, Runx2, BSP, and OCN). All target gene expression results were normalized to $\beta$-actin.

2.12. Statistical Analysis. Data was collected from three separate experiments and shown as the mean \pm standard deviation (SD). The statistical differences were analyzed using one-way analysis of variance (ANOVA) with SPSS 17.0. $p<0.05$ was considered to be statistically significant.

\section{Results and Discussion}

3.1. The Effect of Size and Concentration of AuNPs on hPDLCs Proliferation. Because of their similarity to the nanostructured nature of microenvironment, AuNPs have attracted broad attention in tissue engineering studies. AuNPs like other nanomaterials have high surface area to volume ratio and have the ability to traverse biological barriers and even enter the cell nuclei, affecting the cellular processes such as proliferation and differentiation [12].

Studies have shown that the effects of AuNPs on cells are closely related to the size and concentration of the particles. $\mathrm{Lu}$ et al. demonstrated that $34 \mathrm{~nm}$ gold nanoparticle at a low concentration could enhance the growth of keratinocyte. 
TABLE 1: Primers for quantitative real-time PCR analysis.

\begin{tabular}{|c|c|c|}
\hline Gene & Product size (bp) & Sequences \\
\hline \multirow{2}{*}{ COL-1 } & \multirow{2}{*}{103} & Forward: $5^{\prime}$-GCTCCTCTTAGGGGCCACT-3' \\
\hline & & Reverse: 5'-CCACGTCTCACCATTGGG-3' \\
\hline \multirow{2}{*}{ Runx2 } & \multirow{2}{*}{173} & Forward: 5'-TGTTCTCTGATCGCCTCAGTG-3' \\
\hline & & Reverse: $5^{\prime}$-CCTGGGATCTGTAATCTGACTCT-3' \\
\hline \multirow{2}{*}{ BSP } & \multirow{2}{*}{151} & Forward: 5' -CAGGGAGGCAGTGACTCTTC-3' \\
\hline & & Reverse: 5'-AGTGTGGAAAGTGTGGCGTT-3’ \\
\hline \multirow{2}{*}{ OCN } & \multirow{2}{*}{134} & Forward: $5^{\prime}$-AGCAAGAAACTCTTCCAAGCAA- $3^{\prime}$ \\
\hline & & Reverse: $5^{\prime}$-GTGAGATTCGTCAGATTCATCCG-3' \\
\hline \multirow{2}{*}{$\beta$-actin } & \multirow{2}{*}{108} & Forward: $5^{\prime}$-TTGCTGACAGGATGCAGAAG-3' \\
\hline & & Reverse: $5^{\prime}$-TAGAGCCACCAATCCACACA-3' \\
\hline
\end{tabular}

When the gold nanoparticles exceeded $10 \mathrm{ppm}$, the cytotoxic effect was significantly increased [14].

Their other study showed that $24 \mathrm{~nm}$ gold colloid immobilized onto porcine hepatocytes resulted in a hepatocytes/ gold colloid interface, which could efficiently and rapidly facilitate the proliferation of hepatocytes [15]. Lu et al. reported gold nanoparticles $1-2 \mathrm{~nm}$ in size, which were highly toxic, and both smaller gold compounds (Tauredon) and larger $15 \mathrm{~nm}$ gold colloids, which were comparatively nontoxic, irrespective of the cell type tested [14]. Yao et al. reported that a low concentration $(5.0 \mathrm{ppm})$ of $34 \mathrm{~nm}$ gold nanoparticles could most effectively stimulate keratinocytes proliferation [16]. Yi et al. reported that AuNPs of $30 \mathrm{~nm}$ in diameter at a concentration of $10^{-11} \mathrm{ppm}$ promoted the proliferation of MC3T3-E1 cells most effectively [17]. AuNPs size is a key factor that affects cell adhesion and penetration. The smaller particles inside the cell, which are more stable against aggregation, caused less cell cytotoxicity, whereas the larger particles inside the cell, which are liable to form aggregates, caused more serious cytotoxicity. Cui et al. confirmed this hypothesis through experiments. If the AuNP aggregates are larger than the receptor-mediated endocytosis threshold, the aggregates cannot enter the cell and are nonspecifically adsorbed onto the HeLa cell surface. These large aggregates do not cause cytotoxicity but instead promote the growth of the HeLa cells [18].

In our research, MTS method was used to detect the effect of four sizes of AuNPs in six different concentrations on the proliferation of hPDLCs. The results showed that, compared with the control group, $60 \mathrm{~nm}$ AuNPs could promote cell proliferation and at a concentration of $56 \mu \mathrm{M}$ could promote the proliferation of hPDLCs effectively. The other three sizes of AuNPs had cytotoxic effects on hPDLCs (Figures 1(a)-1(e)).

3.2. The Effect of AuNPs on hPDLSCs Proliferation. Due to the limited resource and the important role of PDLSCs in tissue engineering [3-5, 19-23], how to proliferate PDLSCs rapidly in vitro is a key problem to be solved. hPDLSCs were treated with $60 \mathrm{~nm}$ AuNPs $(56 \mu \mathrm{M})$ (Figure 1(f)). hPDLSCs without AuNPs were used as negative control. Viability of hPDLSCs was evaluated by MTS assay. Two groups of cells grew slowly at 6,12, and 24 hours. At 48 and 72 hours, AuNPs promoted proliferation of hPDLSCs effectively. After 24 hours of culture in AuNPs containing medium, the AuNPs were seen as dense and dark black clusters in the cells. The cells showed no differences in morphology after 24 hours of culture (Figure 2).

3.3. Positive Rate of STRO-1 and CD146 in hPDLSCs Treated with AuNPs. The positive rate of STRO-1 was $84.82 \%$ (Figure 3(a)) and CD146 was $84.25 \%$ (Figure 3(b)) in human periodontal ligament stem cells (hPDLSCs) with AuNPs treatment.

3.4. Effect of AuNPs on ALP Staining and ALP Activity. The ALP staining in cells treated with $60 \mathrm{~nm}$ AuNPs $(56 \mu \mathrm{M})$ and control cells was more intense than blank cells (cells cultured with $\alpha$-MEM medium and not treated with AuNPs) (Figure 4).

The ALP activity of the treated groups was slightly increased compared to the untreated control group. Both experimental group and control group showed significantly higher ALP activity than blank group $(p<0.01)$. ALP is a typical ectoenzyme, which acts as a marker of osteogenic differentiation [24]. So, we measured the ALP level by both quantitative and qualitative methods. The results from the ALP activity assay and ALP staining indicated that AuNPs could induce the differentiation of hPDLSCs (Figure 5).

3.5. Effect of AuNPs Treatment on hPDLSCs Osteogenic and Adipogenic Potential Differentiation and Osteogenic Gene Expression. Recent evidence indicated that AuNPs could promote the osteogenic differentiation and inhibit the adipogenic of MSCs $[17,25,26]$. As shown in Figure 6, calcified depositions were found in cells treated with and without AuNPs (Figures 6(a) and 6(b)). The cells incubated with only $\alpha$-MEM medium were negative with the staining (Figure $6(\mathrm{c}))$. Compared with the control group, the mineralized nodules were larger and more compact in the experimental group. To evaluate the osteogenic differentiation of hPDLSCs, the stained calcified mineral was extracted with cetylpyridinium chloride and the mineralization level was quantified by measuring the absorbance at $570 \mathrm{~nm}$. Alizarin red S extracted from hPDLSCs treated with $60 \mathrm{~nm}$ AuNPs at concentrations of $56 \mu \mathrm{M}$ was $129 \pm 19 \%$ and the control group without AuNPs treatment was $100 \pm 15 \%$ (Figure 7(a)). The mineralization in average in AuNPs treated hPDLSCs 

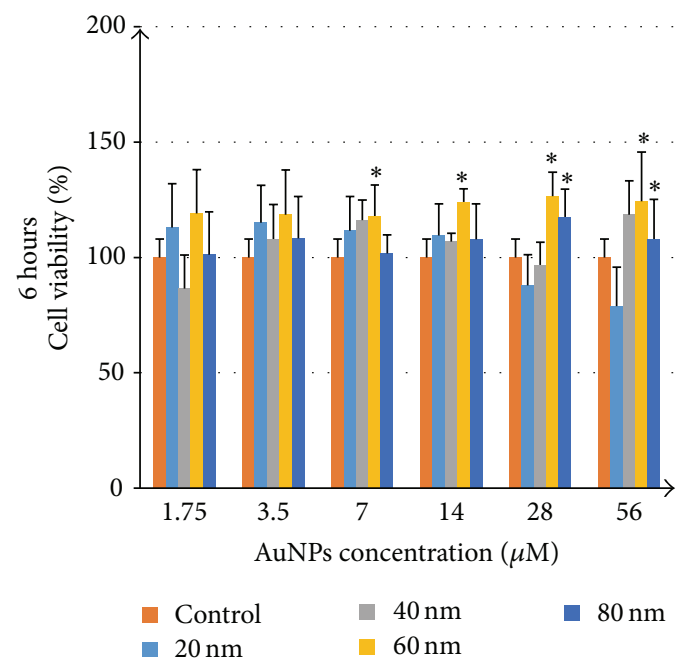

(a)

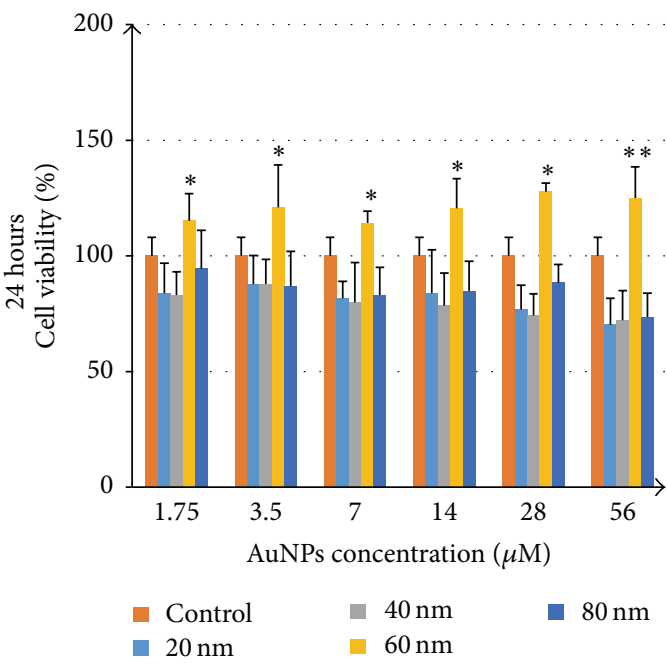

(c)

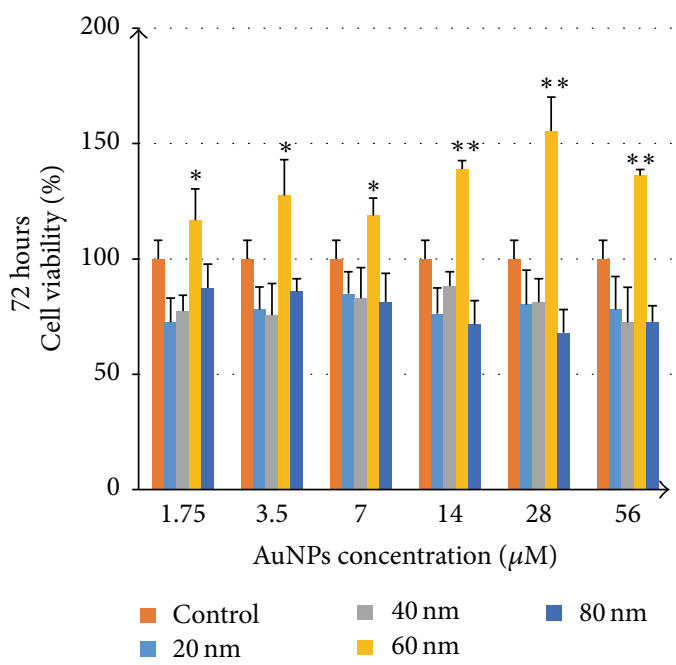

(e)

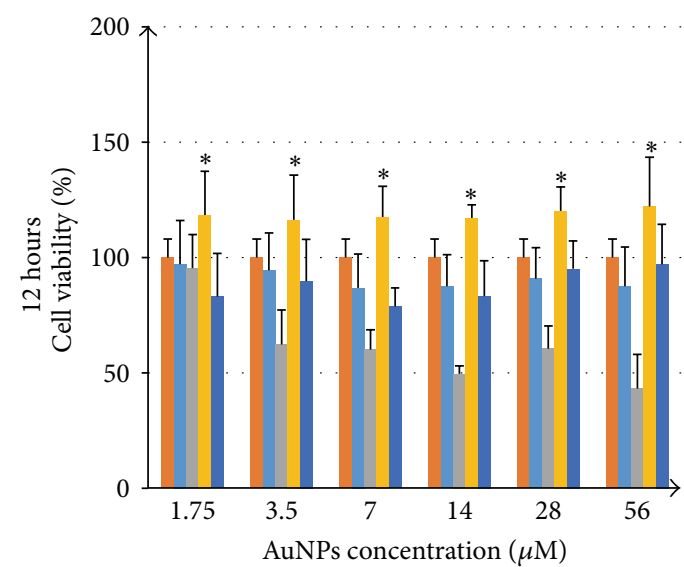

Control $\quad 40 \mathrm{~nm}$

- $20 \mathrm{~nm}$

(b)

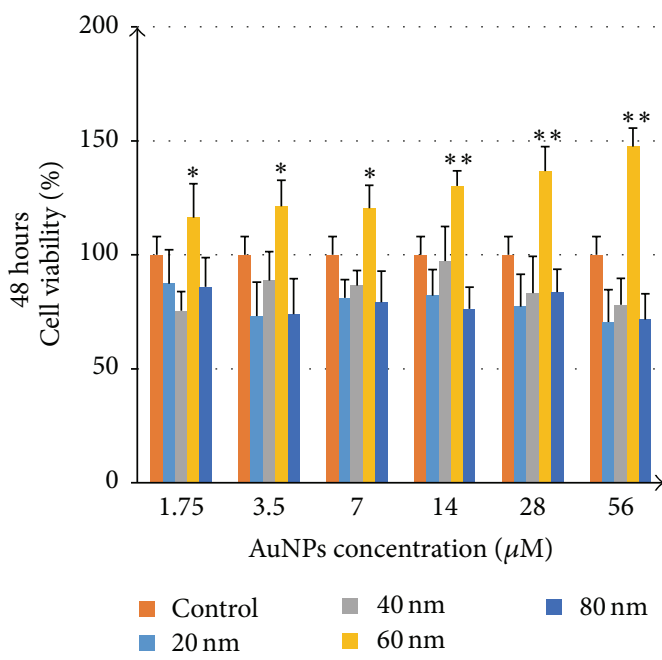

(d)

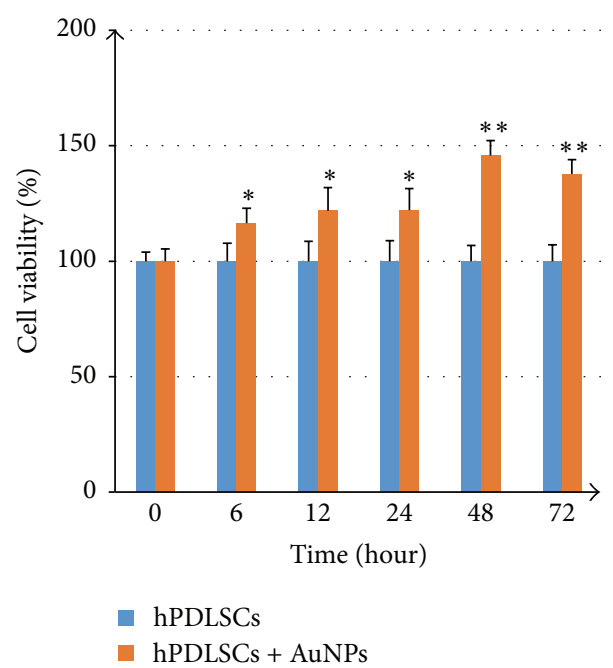

(f)

FIGURE 1: hPDLCs and hPDLSCs proliferation. Viability of hPDLCs incubated with six different concentrations of four sizes AuNPs for 6 (a), 12 (b), 24 (c), 48 (d), and $72 \mathrm{~h}(\mathrm{e}) . n=5$; proliferation and viability of hPDLSCs in the presence of $60 \mathrm{~nm}$ AuNPs $(56 \mu \mathrm{M})$ for 6, 12, 24, 48, and $72 \mathrm{~h}(\mathrm{f}) . n=5$; results are mean \pm SD of five experiments. ${ }^{*}$ Significantly different from control group, $p<0.05$. ${ }^{* *}$ Significantly different from control group, $p<0.01$. 


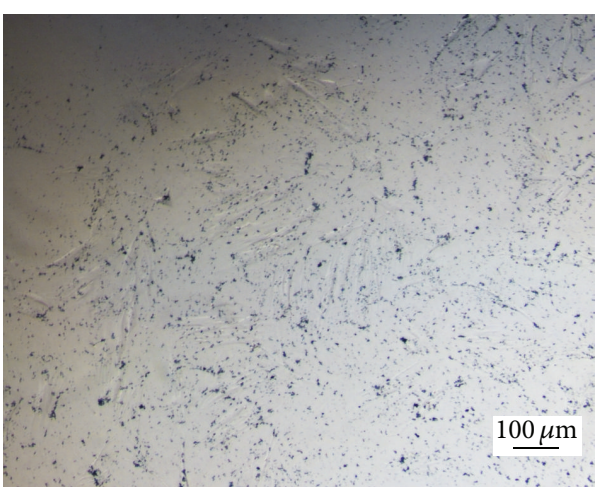

(a)

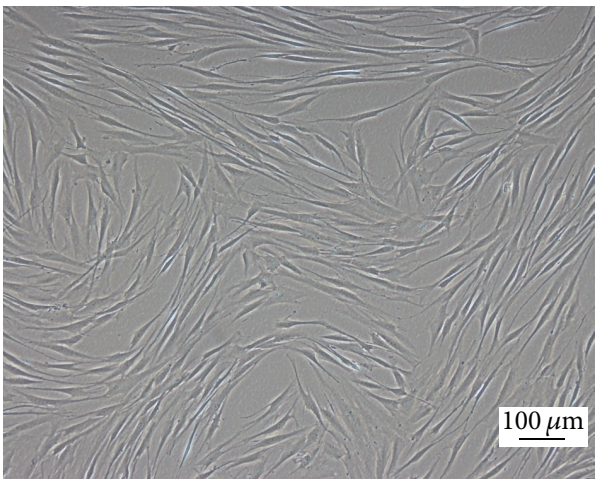

(c)

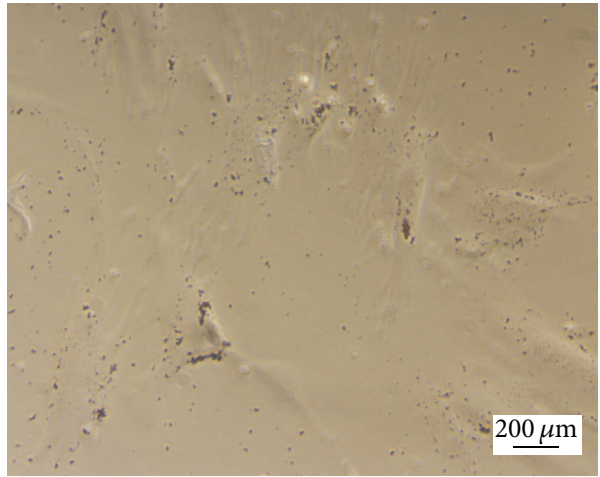

(b)

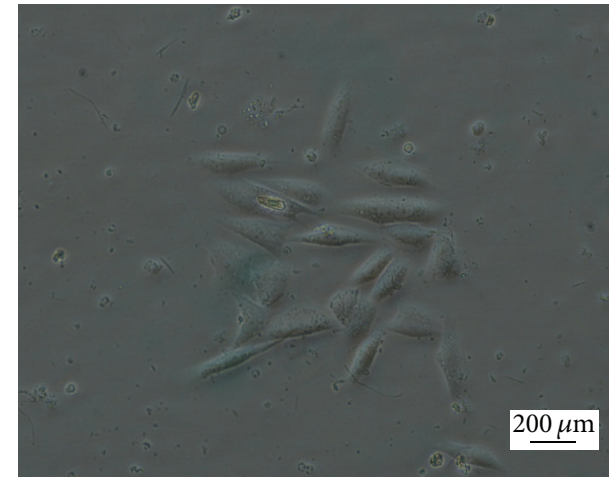

(d)

FIGURE 2: Cell morphology. Representative images of hPDLSCs incubated with $60 \mathrm{~nm}$ AuNPs at $56 \mu \mathrm{M}$ concentrations for $24 \mathrm{~h}$ (a, b) and without AuNPs (c, d). AuNPs were seen as dense and dark black clusters. Cells are spindle or triangular in shape and typically well-spread.

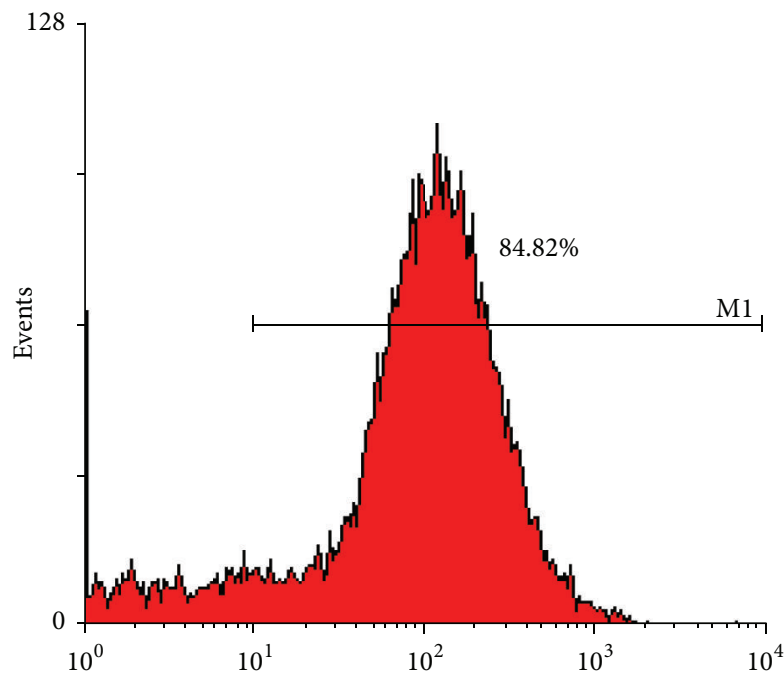

(a)

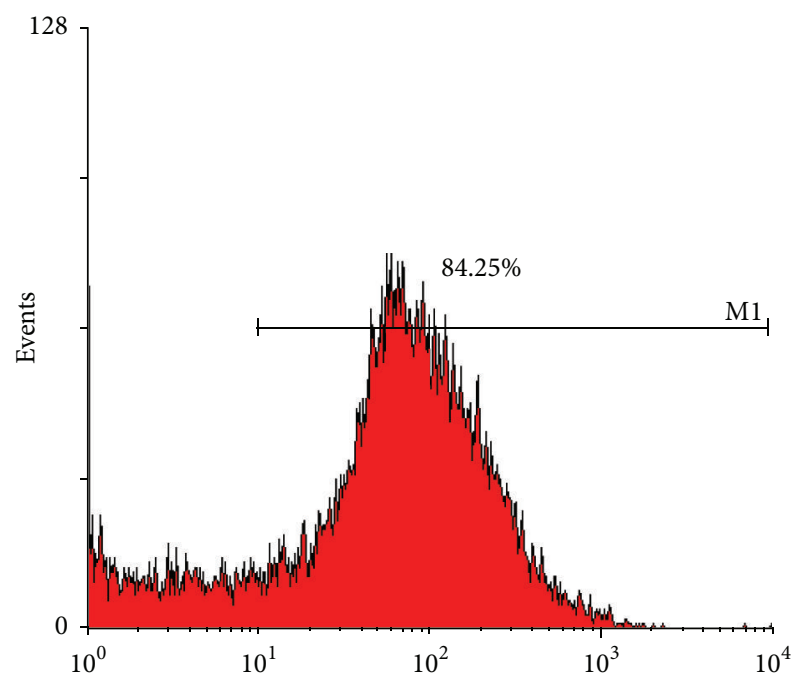

(b)

FIGURE 3: Positive rate of STRO-1 (84.82\%) (a) and CD146 (84.25\%) (b) in hPDLSCs with $60 \mathrm{~nm}$ AuNPs (56 $\mu$ M) treatment for 72 hours.

was higher than the control but without significance $(p>$ $0.05)$.

After adipogenic differentiation for 24 days, cells were stained with Oil red O. As shown in Figure 6, more dyed red lipid droplets were found in the experimental group and the control group under microscope (Figures 6(d) and 6(e)). In the blank group, no staining was found (Figure 6(f)). The stained cytoplasmic lipids in AuNPs treated hPDLSCs were 


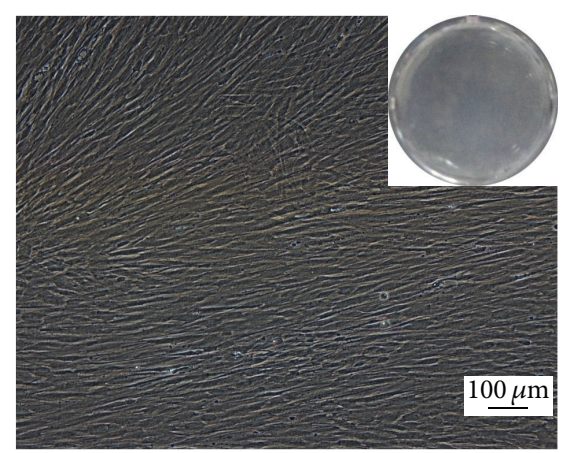

(a)

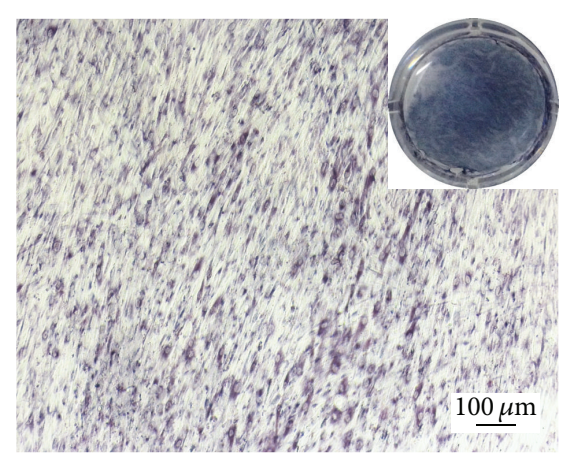

(b)

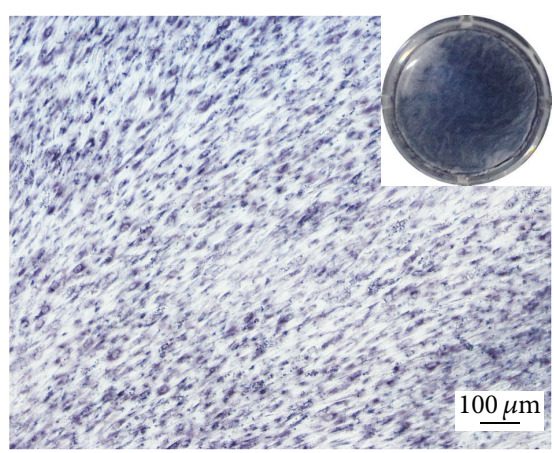

(c)

Figure 4: ALP staining data of hPDLSCs treated with $60 \mathrm{~nm}$ AuNPs $(56 \mu \mathrm{M})$ for 10 days. ALP staining results show that experiment (c) and control group (b) were dark blue, while the blank group was light blue (a).

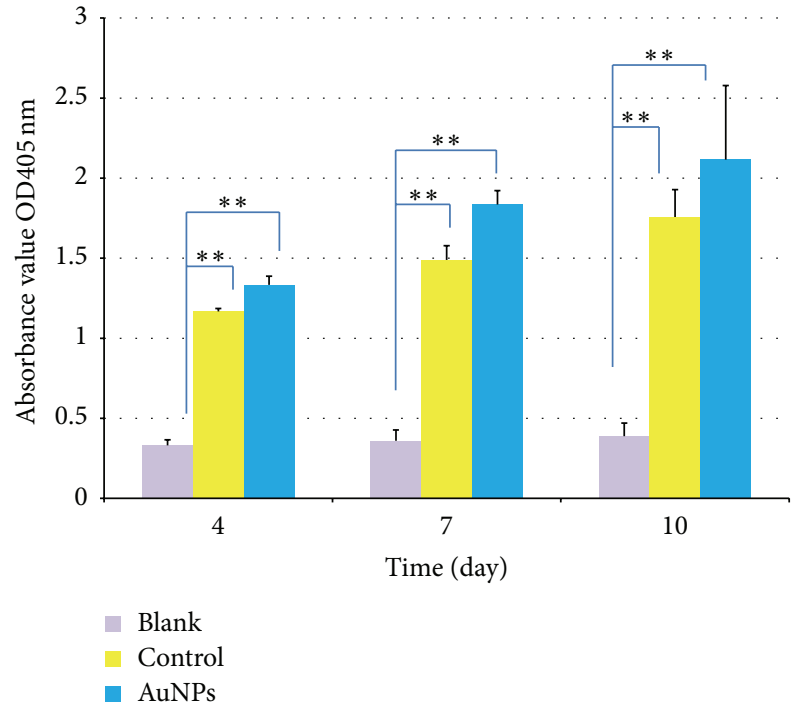

FIgURE 5: ALP activity assay results of cells treated with $60 \mathrm{~nm}$ AuNPs at $56 \mu \mathrm{M}$ concentrations for 4,7 , and 10 days. Results are mean \pm SD of the triplicate experiments; ${ }^{* *} p<0.01$. The ALP activity of the treated groups was slightly increased relative to the untreated control group. Both experiment group and control group significantly are higher than blank group (cell cultured with $\alpha$-MEM medium and not treated with AuNPs).

$115 \pm 14 \%$, while the control group was $100 \pm 12 \%$ (Figure $7(\mathrm{~b}))$. The intracellular lipids content in two groups was not significant $(p>0.05)$.

The results showed that $60 \mathrm{~nm}, 56 \mu \mathrm{M}$ concentration of AuNPs could slightly promote osteoblastic differentiation of hPDLSCs and had no obvious effect on the adipogenic differentiation.

In addition, real-time PCR was used to investigate the expression of the osteogenic genes COL-1, Runx2, BSP, and OCN to determine the effect of AuNPs on the molecular level of hPDLSCs. Several transcription factors have been identified as critical for the regulation of osteoblast differentiation and function, including Runt-related transcription factor 2 (Runx2) and several late marker genes such as osteocalcin
(OCN), collagen type 1 (Col-1), and bone sialoprotein (BSP) [27]. Runx 2 is essential gene for osteoblast differentiation and directly regulates the expression of other osteoblastic specific genes including OCN, osteopontin (OPN), and Col-1. Runx2 knockout mice harbor no bone tissue or osteoblasts, indicating that osteoblast differentiation is completely blocked in the absence of Runx2 [28, 29]. Col-1 is the most abundant protein in bone matrix and usually is formed during the proliferation of osteoblast cells. The expressions of BSP and OCN markers related to calcium deposition in the extracellular matrix during its mineralization stage [28]. In addition, bone extracellular matrix proteins such as osteocalcin $(\mathrm{OCN})$ have important functions during embryonic osteogenesis and are active in the bone remodeling process [30].

COL-1, Runx2, BSP, and OCN are transcription factors which have been identified as critical for the regulation of osteoblast differentiation and function. Figure 8 shows that treatment with AuNPs for four days resulted in an increase in the expression of all four osteogenic genes. The data between the experiment and control group had no statistical differences $(p>0.05)$.

Yi et al. reported that $20 \mathrm{~nm}$ AuNPs at a concentration of $1.0 \mathrm{nM}$ promoted the differentiation of MSCs toward osteoblasts over adipocytes by inducing an enhanced osteogenic transcriptional profile and an attenuated adipogenic transcriptional profile. AuNPs exerted the effects by interacting with the cell membrane and binding with proteins in the cytoplasm, causing mechanical stress on the MSCs to activate p38 mitogen activated protein kinase (MAPK) signaling pathway, which regulates the expression of relevant genes [17]. Choi et al. reported that chitosan-conjugated AuNPs promote the osteogenic differentiation of human adipose-derived mesenchymal stem cells through the Wnt/ $\beta$ catenin signaling pathway [25]. Zhang et al. reported similar conclusion drawn from experiments. They found that AuNPs could significantly promote the proliferation of osteoblasts, enhance the ALP activities, and increase the number of bone nodules and calcium content in vitro. In addition, the expression of BMP-2, Runx-2, OCN, and COL-1 was remarkably upregulated in the presence of AuNPs. It is noteworthy that $20 \mathrm{~nm}$ AuNPs is more potent than $40 \mathrm{~nm}$ AuNPs in 


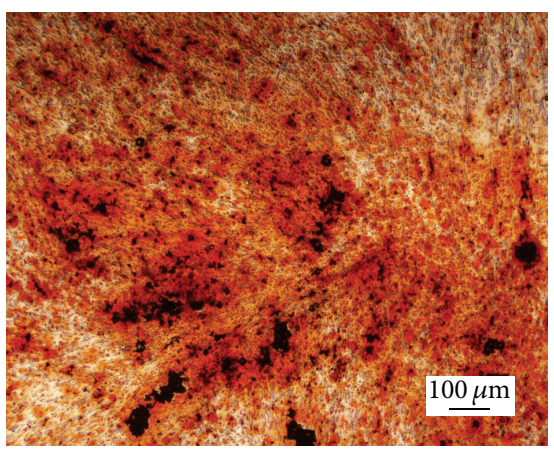

(a)

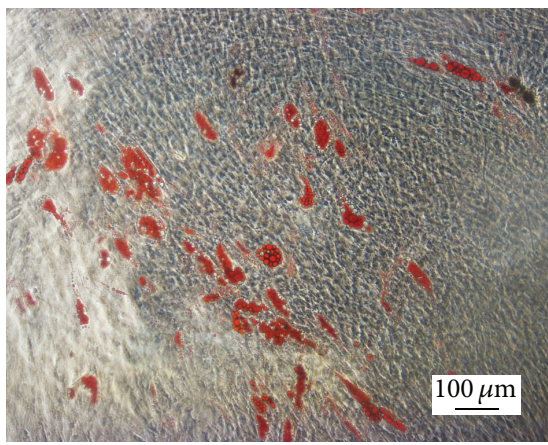

(d)

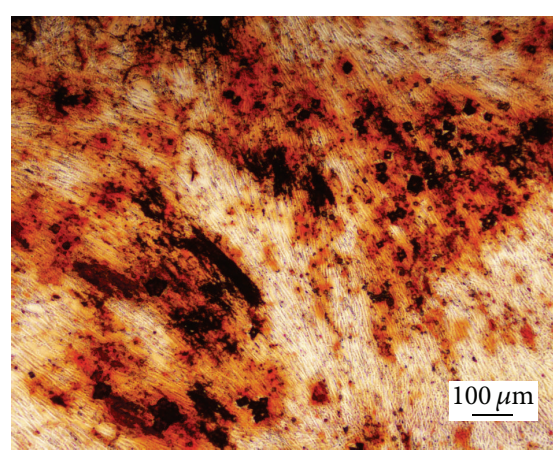

(b)

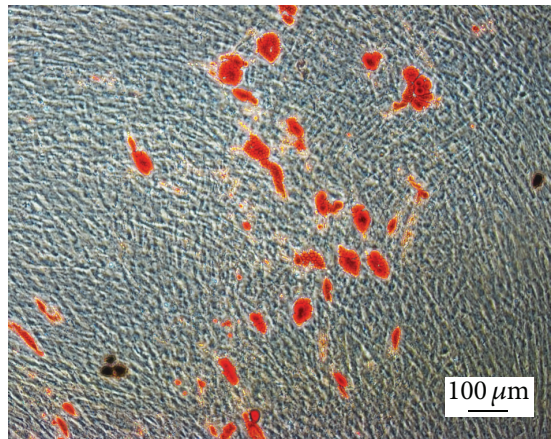

(e)

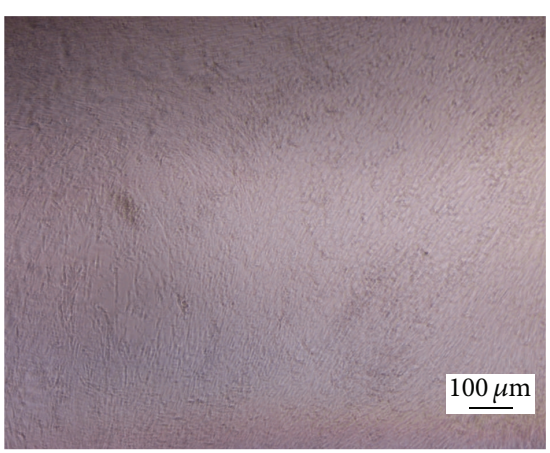

(c)

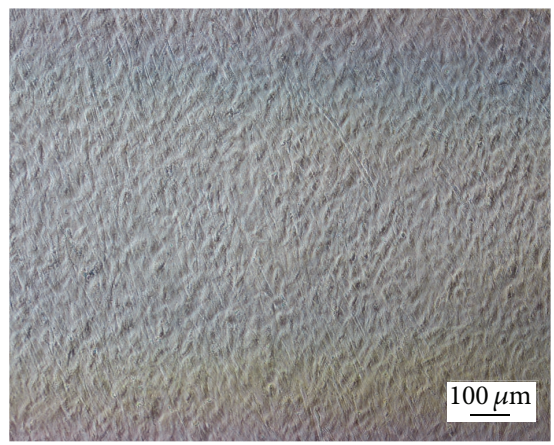

(f)

FIGURE 6: Osteogenic and adipogenic differentiation of hPDLSCs cocultured with AuNPs $(60 \mathrm{~nm}, 56 \mu \mathrm{M})$. Calcified deposition was found in cells treated with and without AuNPs $(\mathrm{a}, \mathrm{b})$ for 28 days. The cells incubated with only $\alpha$-MEM medium were not positive for the staining (c). Compared with the control group, mineralized nodules of the experimental group were larger and more compact. After adipogenic differentiation for 24 days, cells were stained with Oil red O. More dyed red lipid droplets were found in the experimental group (d) and the control group (e) under microscope. In the blank group (f), no staining was found.

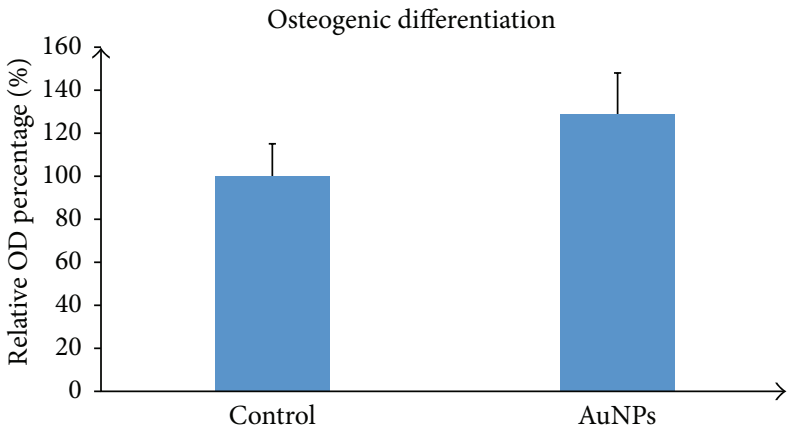

(a)

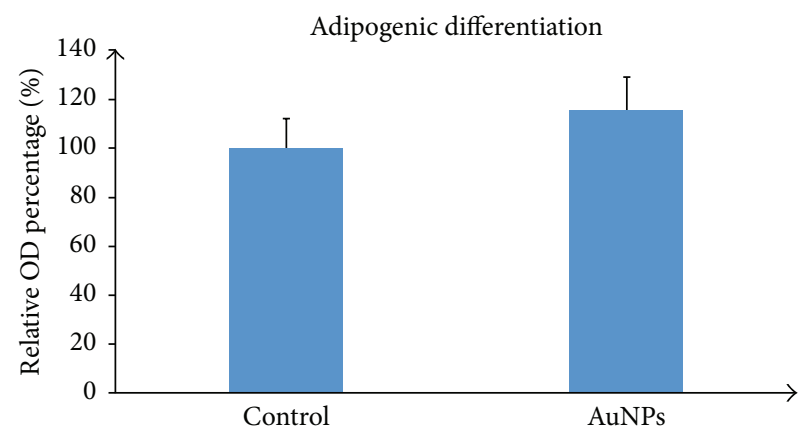

(b)

FIGURE 7: The quantification of Alizarin red staining and Oil red O staining. (a) The relative mineralization rate of AuNPs treated group compared to control group. (b) The intracellular lipids content of hPDLSCs treated with $60 \mathrm{~nm}$ AuNPs compared to the control.

regulating osteoblast activities [26]. Our experimental results showed that AuNPs promoted the osteoblastic differentiation of hPDLSCs, but it showed no significant difference compared with the control group. The reason could be related to the particle size of AuNPs. The one used in the experiment was $60 \mathrm{~nm}$, which is larger than 20 and $40 \mathrm{~nm}$. Other nanomaterials also have effect on MSCs differentiation. Qiu et al. recently reported graphene quantum dots (GQDs), a new type of carbon-based quantum dots, which can enhance the osteogenic differentiation of MSCs through a bone morphogenetic protein and transforming growth factor $-\beta$ relative signaling pathways. Furthermore, GQDs can also promote the adipogenic differentiation of MSCs [31].

\section{Conclusion}

In this study, we investigated the effect of gold nanoparticles on proliferation and differentiation of hPDLSCs. Our 


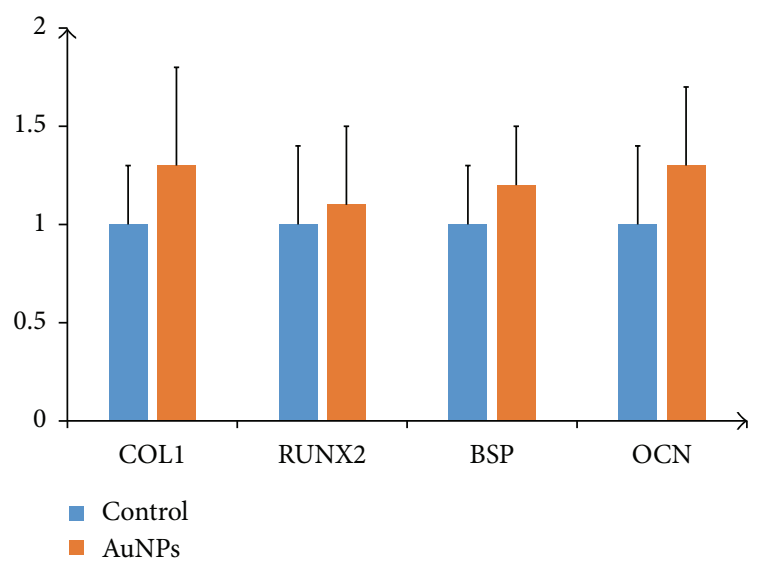

FIgURE 8: The effect of AuNPs on the expression of osteogenic genes. A real-time PCR analysis of the osteogenic marker gene expression in hPDLSCs treated with $60 \mathrm{~nm}$ AuNPs for four days. The relative expression levels of target genes normalized to $\beta$-actin. Data represent mean \pm SD of the triplicate experiments.

results demonstrated that AuNPs with $60 \mathrm{~nm}$ diameter at a concentration of $56 \mu \mathrm{M}$ can promote the proliferation of hPDLSCs and have no significant effect on the differentiation of hPDLSCs.

\section{Competing Interests}

No potential competing interests were disclosed.

\section{Acknowledgments}

The authors gratefully acknowledge the financial supports by the National Science Foundation of China under Grant no. 81271152 . Useful suggestions given by Dr. Kaili Lin of Tongji University are also to be acknowledged. This work was supported by the National Natural Science Foundation of China (no. 81271152).

\section{References}

[1] R. C. Page, S. Offenbacher, H. E. Schroeder, G. J. Seymour, and K. S. Kornman, "Advances in the pathogenesis of periodontitis: summary of developments, clinical implications and future directions," Periodontology 2000, vol. 14, no. 1, pp. 216-248, 1997.

[2] B. M. Seo, M. Miura, S. Gronthos et al., "Investigation of multipotent postnatal stem cells from human periodontal ligament," Lancet, vol. 364, no. 9429, pp. 149-155, 2004.

[3] S.-H. Kim, K.-H. Kim, B.-M. Seo et al., "Alveolar bone regeneration by transplantation of periodontal ligament stem cells and bone marrow stem cells in a canine peri-implant defect model: a pilot study," Journal of Periodontology, vol. 80, no. 11, pp. 18151823, 2009.

[4] D. Fang, B.-M. Seo, Y. Liu et al., "Transplantation of mesenchymal stem cells is an optimal approach for plastic surgery," STEM CELLS, vol. 25, no. 4, pp. 1021-1028, 2007.

[5] J. Xu, W. Wang, Y. Kapila, J. Lotz, and S. Kapila, "Multiple differentiation capacity of STRO $-1^{+} / \mathrm{CD} 146^{+}$PDL mesenchymal progenitor cells," Stem Cells and Development, vol. 18, no. 3, pp. 487-496, 2009.
[6] V. Vacanti, E. Kong, G. Suzuki, K. Sato, J. M. Canty, and T. Lee, "Phenotypic changes of adult porcine mesenchymal stem cells induced by prolonged passaging in culture," Journal of Cellular Physiology, vol. 205, no. 2, pp. 194-201, 2005.

[7] T. Itaya, H. Kagami, K. Okada et al., "Characteristic changes of periodontal ligament-derived cells during passage," Journal of Periodontal Research, vol. 44, no. 4, pp. 425-433, 2009.

[8] Y. Cheng, J. D. Meyers, A.-M. Broome, M. E. Kenney, J. P. Basilion, and C. Burda, "Deep penetration of a PDT drug into tumors by noncovalent drug-gold nanoparticle conjugates," Journal of the American Chemical Society, vol. 133, no. 8, pp. 2583-2591, 2011.

[9] E. Hutter and D. Maysinger, "Gold nanoparticles and quantum dots for bioimaging," Microscopy Research and Technique, vol. 74, no. 7, pp. 592-604, 2011.

[10] R. Arvizo, R. Bhattacharya, and P. Mukherjee, "Gold nanoparticles: opportunities and challenges in nanomedicine," Expert Opinion on Drug Delivery, vol. 7, no. 6, pp. 753-763, 2010.

[11] C. Posch, A. Latorre, M. B. Crosby et al., "Detection of GNAQ mutations and reduction of cell viability in uveal melanoma cells with functionalized gold nanoparticles," Biomedical Microdevices, vol. 17, no. 1, 2015.

[12] A. M. Alkilany, S. E. Lohse, and C. J. Murphy, "The gold standard: Gold nanoparticle libraries to understand the nano-bio interface," Accounts of Chemical Research, vol. 46, no. 3, pp. 650661, 2013.

[13] R. A. Sperling, P. R. Gil, F. Zhang, M. Zanella, and W. J. Parak, "Biological applications of gold nanoparticles," Chemical Society Reviews, vol. 37, no. 9, pp. 1896-1908, 2008.

[14] S. Lu, D. Xia, G. Huang, H. Jing, Y. Wang, and H. Gu, “Concentration effect of gold nanoparticles on proliferation of keratinocytes," Colloids and Surfaces B: Biointerfaces, vol. 81, no. 2, pp. 406-411, 2010.

[15] H.-Y. Gu, Z. Chen, R.-X. Sa et al., “The immobilization of hepatocytes on $24 \mathrm{~nm}$-sized gold colloid for enhanced hepatocytes proliferation," Biomaterials, vol. 25, no. 17, pp. 3445-3451, 2004.

[16] Y. Yao, X. Shi, and F. Chen, "The effect of gold nanoparticles on the proliferation and differentiation of murine osteoblast: a study of MC3T3-E1 cells in vitro," Journal of Nanoscience and Nanotechnology, vol. 14, no. 7, pp. 4851-4857, 2014.

[17] C. Yi, D. Liu, C.-C. Fong, J. Zhang, and M. Yang, "Gold nanoparticles promote osteogenic differentiation of mesenchymal stem cells through p38 MAPK pathway," ACS Nano, vol. 4, no. 11, pp. 6439-6448, 2010.

[18] W. Cui, J. Li, Y. Zhang, H. Rong, W. Lu, and L. Jiang, "Effects of aggregation and the surface properties of gold nanoparticles on cytotoxicity and cell growth," Nanomedicine, vol. 8, no. 1, pp. 46-53, 2012.

[19] F. Feng, K. Akiyama, Y. Liu et al., "Utility of PDL progenitors for in vivo tissue regeneration: a report of 3 cases," Oral Diseases, vol. 16, no. 1, pp. 20-28, 2010.

[20] T. Akizuki, S. Oda, M. Komaki et al., "Application of periodontal ligament cell sheet for periodontal regeneration: a pilot study in beagle dogs," Journal of Periodontal Research, vol. 40, no. 3, pp. 245-251, 2005.

[21] J.-Y. Park, S. H. Jeon, and P.-H. Choung, "Efficacy of periodontal stem cell transplantation in the treatment of advanced periodontitis," Cell Transplantation, vol. 20, no. 2, pp. 271-285, 2011.

[22] Y. Liu, Y. Zheng, G. Ding et al., "Periodontal ligament stem cell-mediated treatment for periodontitis in miniature swine," STEM CELLS, vol. 26, no. 4, pp. 1065-1073, 2008. 
[23] K. Washio, T. Iwata, M. Mizutani et al., "Assessment of cell sheets derived from human periodontal ligament cells: a pre-clinical study," Cell and Tissue Research, vol. 341, no. 3, pp. 397-404, 2010.

[24] D. Liu, C. Yi, D. Zhang, J. Zhang, and M. Yang, "Inhibition of proliferation and differentiation of mesenchymal stem cells by carboxylated carbon nanotubes," ACS Nano, vol. 4, no. 4, pp. 2185-2195, 2010.

[25] S. Y. Choi, M. S. Song, P. D. Ryu, A. T. N. Lam, S.-W. Joo, and S. Y. Lee, "Gold nanoparticles promote osteogenic differentiation in human adipose-derived mesenchymal stem cells through the Wnt/ $\beta$-catenin signaling pathway," International Journal of Nanomedicine, vol. 10, pp. 4383-4392, 2015.

[26] D. Zhang, D. Liu, J. Zhang, C. Fong, and M. Yang, "Gold nanoparticles stimulate differentiation and mineralization of primary osteoblasts through the ERK/MAPK signaling pathway," Materials Science and Engineering: C, vol. 42, pp. 70-77, 2014.

[27] J. Skillington, L. Choy, and R. Derynck, "Bone morphogenetic protein and retinoic acid signaling cooperate to induce osteoblast differentiation of preadipocytes," Journal of Cell Biology, vol. 159, no. 1, pp. 135-146, 2002.

[28] T. Komori, "Requisite roles of Runx2 and Cbfb in skeletal development," Journal of Bone and Mineral Metabolism, vol. 21, no. 4, pp. 193-197, 2003.

[29] F. Otto, A. P. Thornell, T. Crompton et al., "Cbfal, a candidate gene for cleidocranial dysplasia syndrome, is essential for osteoblast differentiation and bone development," Cell, vol. 89, no. 5, pp. 765-771, 1997.

[30] J. Li, X. Mou, J. Qiu et al., "Surface charge regulation of osteogenic differentiation of mesenchymal stem cell on polarized ferroelectric crystal substrate," Advanced Healthcare Materials, vol. 4, no. 7, pp. 998-1003, 2015.

[31] J. Qiu, D. Li, X. Mou et al., "Effects of graphene quantum dots on the self-renewal and differentiation of mesenchymal stem cells," Advanced Healthcare Materials, vol. 5, no. 6, pp. 702-710, 2016. 

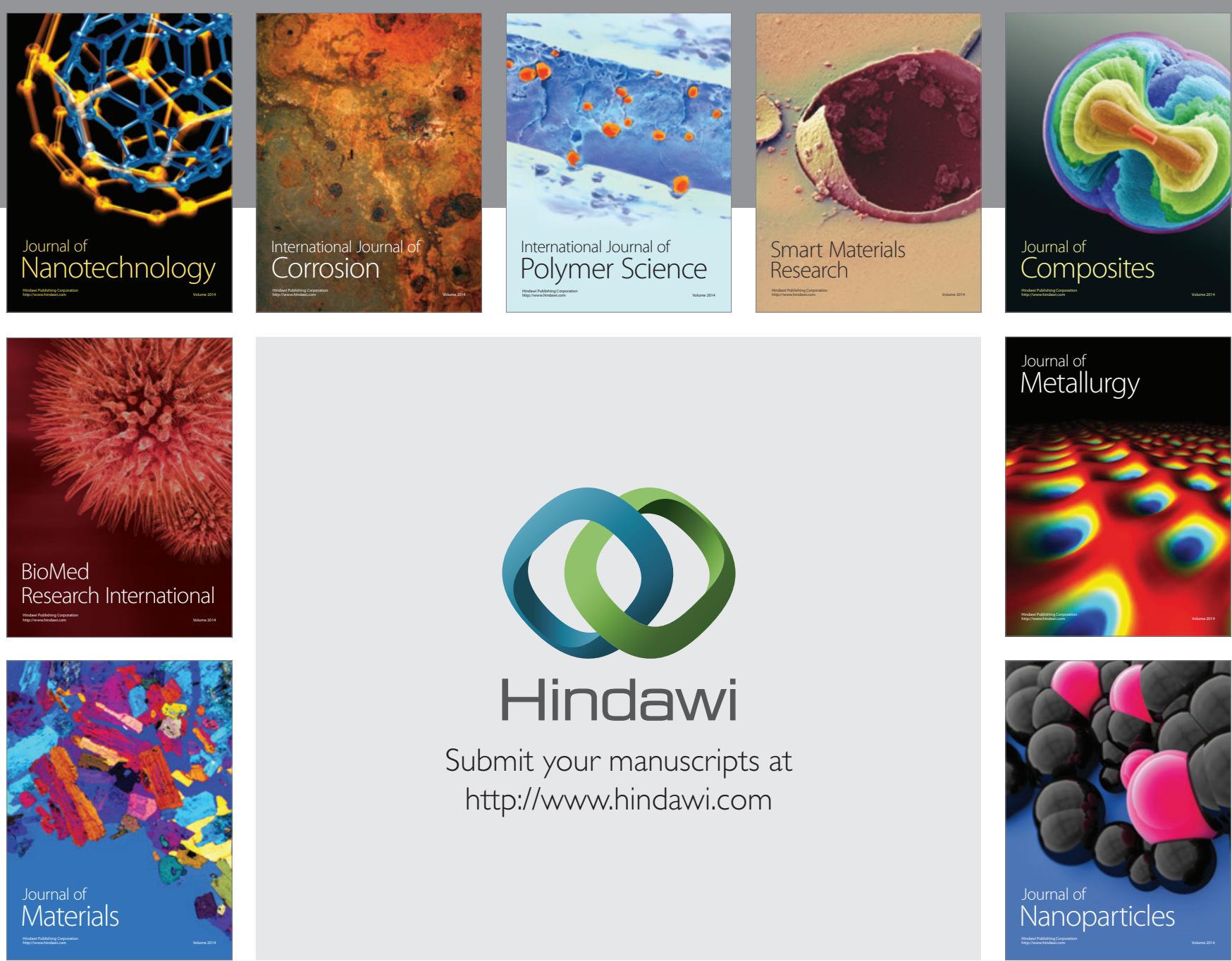

\section{Hindawi}

Submit your manuscripts at

http://www.hindawi.com

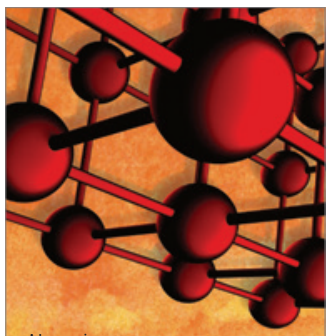

Materials Science and Engineering
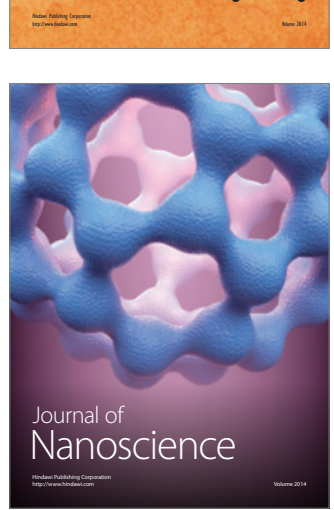
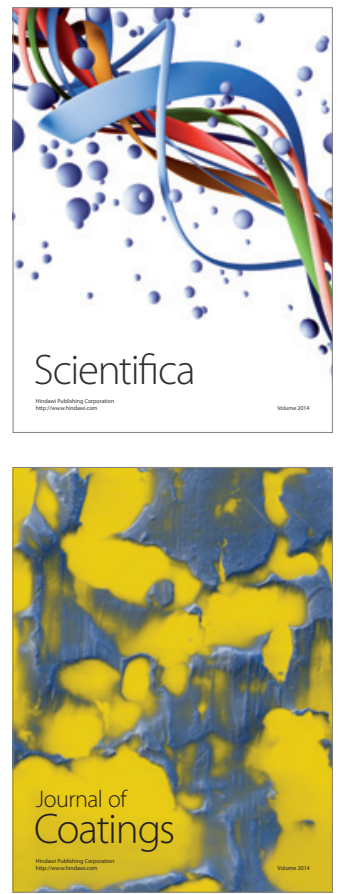
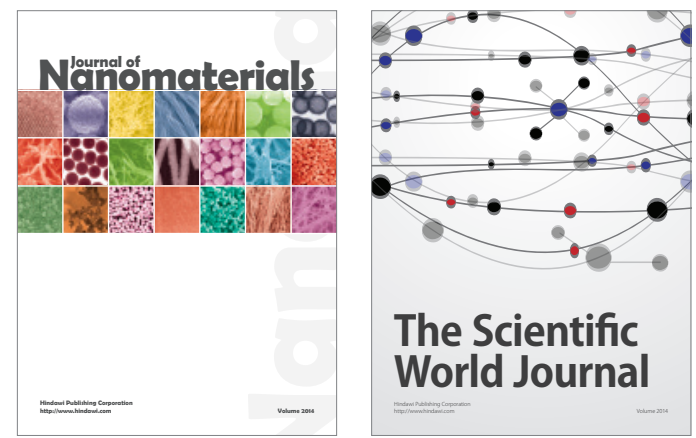

The Scientific World Journal
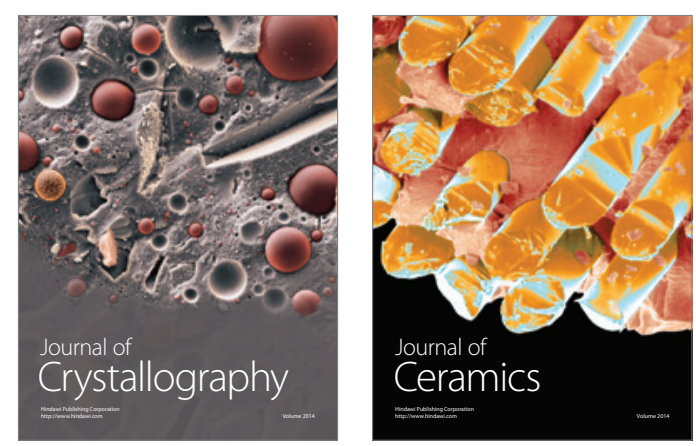
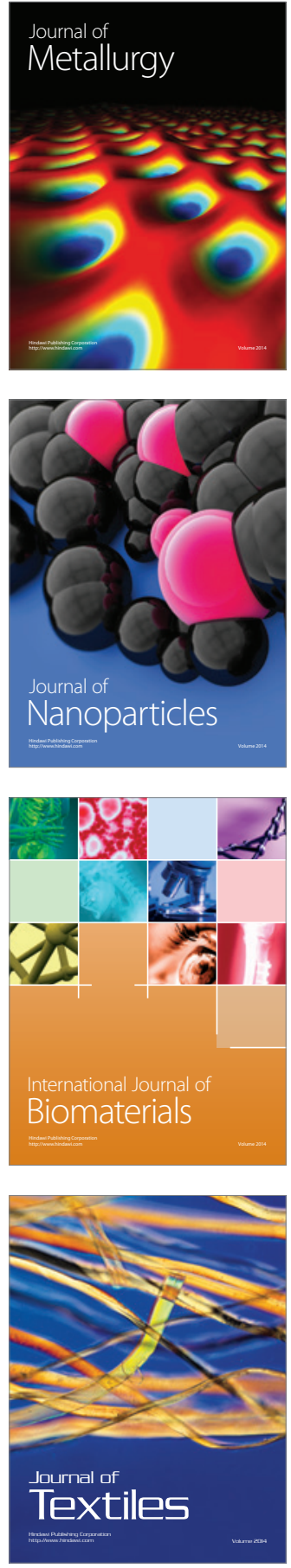